\title{
Evidências de validade da Escala de Experiências Dissociativas em amostra não clínica ${ }^{1}$
}

\author{
Everton de Oliveira Maraldi², Wellington Zangari \\ Universidade de São Paulo, São Paulo-SP, Brasil
}

\section{RESUMO}

O objetivo deste artigo é apresentar as propriedades psicométricas, a estrutura fatorial e evidências de validade convergente da versão brasileira da Escala de Experiências Dissociativas (DES) em uma amostra não clínica. Participaram deste estudo 1450 respondentes (67,9\% homens) com 18 anos de idade ou mais $(M=29,32 ; D P=11,27)$, de vários estados brasileiros, que integraram um survey online sobre o papel das experiências dissociativas nas práticas religiosas e a relação com variáveis psicopatológicas e sociodemográficas. A análise fatorial exploratória indicou que a melhor solução seria trifatorial, tendo os fatores sido nomeados como: absorção, despersonalização-desrealização e amnésia. A versão brasileira da DES denotou consistência interna satisfatória. Também correlacionou positivamente com variáveis com as quais se esperava que correlacionasse, como sintomas conversivos e de somatização e relatos de experiências místico-religiosas. Pessoas com escore igual ou acima de 30 apresentaram média significativamente maior em abuso emocional, abuso físico e abuso sexual.

Palavras-chave: dissociação; precisão do teste; análise fatorial; correlação estatística.

\section{ABSTRACT - Validity evidence of the Dissociative Experiences Scale in a nonclinical sample}

The aim of this paper is to present the psychometric properties, factor structure and evidence of convergent validity of the Brazilian version of the Dissociative Experiences Scale (DES) in a non-clinical sample. The study included 1,450 respondents (67.9\% male), 18 and older $(M=29.32 ; S D=11.27)$, from several Brazilian states, who joined an online survey about the role of dissociative experiences in religious practices and their relation to sociodemographic and psychopathological variables. The exploratory factor analysis indicated that the best solution would be tri-factorial. The factors were named as absorption, depersonalization/derealization and amnesia. The Brazilian DES denoted satisfactory internal consistency. The DES also correlated positively with expected variables, as measures of somatization and conversion symptoms, and reports of religious experiences. Participants with a score greater than or equal to 30 in the DES presented significantly higher scores in emotional abuse, physical abuse and sexual abuse.

Keywords: dissociation; measurement precision; factor analysis; statistical correlation.

\section{RESUMEN - Evidencias de validez de la Escala de Experiencias Disociativas em muestra no clinica}

El objetivo de este artículo es presentar en una muestra no clínica, las propiedades psicométricas, la estructura factorial y evidencias de validez convergente de la versión brasileña de la Escala de Experiencias Disociadas (DES). Participaron 1.450 encuestados (67,9\% hombres), con 18 o más años de edad $(M=29.32, S D=11.27)$, de varios estados brasileños, que integraron un levantamiento online sobre el papel de las experiencias disociativas en las prácticas religiosas, y su relación con variables sociodemográficas y psicopatológicas. El análisis factorial exploratorio indicó que la mejor solución sería trifactorial, siendo esos factores : absorción, despersonalización / desrealización y amnesia. La versión brasileña de la DES indica consistencia interna satisfactoria. La DES también se correlacionó positivamente con las variables con las cuales se esperaba que se correlacionase, como síntomas de conversión y de somatización, y relatos de experiencias místico-religiosas. Las personas con resultados igual o mayores de 30 en la DES presentaron media significativamente mayor en abuso emocional, abuso físico y abuso sexual.

Palabras clave: disociación; precisión del test; análisis factorial; correlación estadística.

A dissociação pode ser definida como uma perda temporária ou persistente de informação ou controle sobre processos mentais ou psicomotores que se acham ordinariamente sob o domínio voluntário da consciência, do repertório comportamental usual ou do autoconceito (Cardeña \& Carlson 2011). O fenômeno da dissociação é multifacetado e pode variar da absorção imaginativa, passando pelos estados de transe, amnésia e fuga dissociativas (com a formação de lacunas mais ou menos significativas na memória), até a alegada divisão

Agradecimentos à FAPESP - Fundação de Amparo à Pesquisa do Estado de São Paulo, pelo financiamento concedido para a realização da presente pesquisa (Processo n²011/05666-1)

2 Endereço para correspondência: Avenida Prof. Mello Moraes, 1721, Departamento de Psicologia Social e do Trabalho, Cidade Universitária, São Paulo-SP. Tel.: (11) 3091-4460. E-mail: evertonom@usp.br 
do Eu em identidades distintas e conflitantes, como no chamado transtorno dissociativo de identidade (TDI). Todavia, em geral, considera-se que as experiências dissociativas nem sempre são patológicas, podendo decorrer de uma série de atividades humanas culturalmente aceitas e possivelmente adaptativas, como, por exemplo, certas práticas religiosas e espirituais (experiências mediúnicas, dons do espírito santo, etc.) e práticas esportivas, como experiências de absorção e alheamento experimentadas por maratonistas (Alvarado, 2005; Krippner, 1997; Maraldi, 2014).

Definições clínicas mais recentes se referem a duas modalidades básicas de dissociação: a compartimentalização e o alheamento. A dissociação enquanto compartimentalização envolve fenômenos em que se observa o isolamento funcional de conteúdos mentais diversos (sensações, lembranças, funções psicomotoras, etc.), os quais passam a agir com maior ou menor autonomia em relação à consciência. A compartimentalização incluiria a amnésia dissociativa, os fenômenos conversivos e outros exemplos de dissociação somatoforme. Já o alheamento corresponde a experiências de ausência e "alienação" frente a si mesmo e ao ambiente imediato, em que ocorreriam alterações da consciência. Em alguns casos, como nas experiências fora do corpo e na despersonalização-desrealização, o indivíduo se vê aparentemente "de fora", como se os eventos acontecessem com outra pessoa, ou relata alterações significativas na imagem corporal e na percepção do ambiente. Aqui também se incluiria a dissociação peritraumática, isto é, a dissociação que se dá durante ou próxima de um evento traumático (Spitzer, Barnow, Freyberger, \& Grabe, 2006).

Historicamente, o conceito de dissociação é herdeiro da noção mais ampla e mais antiga de histeria (Kihlstrom, 1994). Alguns dos autores pioneiros da Psicologia e da Psiquiatria, a exemplo de Charcot, Janet e o próprio Freud (no início), concebiam que a histeria resultava, em parte, de experiências traumáticas, às vezes ocorridas em tenra infância. Essa ideia ficou conhecida, na literatura mais recente sobre dissociação, como "o modelo do trauma" ou trauma model (Dalenberg et al., 2012) o qual recebeu acaloradas discussões (Dalenberg et al., 2014; Lynn et al., 2014). O modelo do trauma defende que a dissociação constitui uma reação a experiências de abuso e negligência, sobretudo, na infância, representando um aspecto importante da resposta biológica à ameaça $\mathrm{e}$ ao perigo, o qual permite a automatização do comportamento, analgesia, despersonalização e isolamento funcional de experiências catastróficas (amnésia). Todavia, em excesso, tal mecanismo acabaria por gerar desvantagens posteriores, pelo próprio prejuízo causado às funções cognitivas e emocionais que teve de atingir para evitar ou diminuir o nível de sofrimento e o impacto do trauma. Há controvérsias, porém, quanto à validade desse modelo para amostras não clínicas (Irwin, 1999; Nijenhuis \& Van der Hart, 2011).
A partir da década de 1980, a pesquisa da dissociação passou a adotar uma perspectiva claramente mais psicométrica e diagnóstica, em contraste com os interesses mais teóricos que preocupavam os autores pioneiros do campo, no final do século XIX. As mudanças nas categorias diagnósticas dos manuais psiquiátricos (DSM e CID) levaram ao surgimento de novas formas de classificação da dissociação em suas modalidades patológicas. $\mathrm{O}$ reconhecimento de uma insuficiência do guarda-chuva mais amplo da histeria ocasionou sua fragmentação em patologias diversas que representavam os esforços dos clínicos em buscarem diagnósticos mais precisos e confiáveis. Uma vez desprendida da histeria e da esquizofrenia, a dissociação adquire lugar próprio entre as demais classificações psiquiátricas (Khilstrom, 1994; Van der Hart \& Dorahy, 2009).

Buscando instrumentos de avaliação padronizados, os pesquisadores da dissociação desenvolveram escalas e inventários para fins de rastreio e mesmo diagnóstico dos transtornos dissociativos. A escala de experiências dissociativas (Dissociative Experiences Scale DES; Bernstein \& Putnam, 1986; Carlson \& Putnam, 1993) é o questionário mais utilizado mundialmente para rastrear e quantificar fenômenos dissociativos. A escala contém 28 questões relativas a experiências que se pode ter no dia a dia, quando não se está sob o efeito de drogas ou álcool. Até o momento, a DES já foi aplicada em aproximadamente 2.000 estudos e traduzida para vários idiomas. Além de apresentar boas propriedades psicométricas e forte correlação estatística com outros questionários sobre dissociação, a DES não revelou associação com desejabilidade social (Dalenberg et al., 2012; Van Ijzendoorn \& Schuengel, 1996). Já em relação ao perfil sociodemográfico dos respondentes, algumas pesquisas indicaram que as mulheres tendem a pontuar mais do que os homens, e haveria correlação negativa entre a DES e idade (Irwin, 1999). Por sua vez, indivíduos com menor inteligência e escolaridade tendem a pontuar mais (Van Ijzendoorn \& Schuengel, 1996).

Segundo Nijenhuis (2000), a DES engloba questões sobre fenômenos dissociativos que são de cunho mais cognitivo do que somatoforme, como é o caso da absorção, da desrealização-despersonalização e das alterações de memória e de personalidade. Não obstante, é possível encontrar nela algumas perguntas referentes a 1 . sintomas de analgesia e 2 . habilidades motoras ou sociais que o indivíduo pode desempenhar melhor em estado dissociativo. É comum aos pesquisadores verificarem associações positivas entre escalas de somatização e dissociação (Jepsen, Langeland, \& Heir, 2014; Kruesi, Borckhardt, Younger, Nash, \& Shaw, 2004). Sabe-se que a somatização está fortemente vinculada a queixas depressivas e ansiógenas (Simms, Prisciandaro, Krueger, \& Goldberg, 2012), e que o estresse representa um fator subjacente, comum tanto a certas formas de dissociação quanto à somatização e à conversão (Nijenhuis, 2000). 
Nesse sentido, sintomas aparentemente físicos, mas não explicáveis em termos médicos, poderiam constituir uma expressão somática de sofrimento psíquico, possivelmente, mediada pelos efeitos da dissociação (Simms et al., 2012). A despeito das evidências em contrário, a edição mais recente do Manual Diagnóstico e Estatístico de Transtornos Mentais (DSM-V) manteve separadas as classificações da dissociação, da conversão e da somatização (American Psychiatric Association, 2013). Para uma crítica dessa postura, ver Nijenhuis (2014).

Várias análises de fatores foram conduzidas com a DES, mas a conclusão inicial dos autores era de que a escala só avaliaria de modo confiável um único fator geral de dissociação (Carlson \& Putnam, 1993). Contudo, recomendou-se aos pesquisadores para não relatarem apenas médias ou diferenças entre médias grupais, mas igualmente as diferenças entre itens específicos, de forma a se acessar padrões mais sutis de relação entre os dados. Alguns estudos encontraram três subescalas além do escore total (Carlson et al., 1991; Ross, Joshi, \& Currie, 1991), abarcando as dimensões da absorção/envolvimento imaginativo, amnésia e despersonalização/desrealização. Waller, Carlson, e Putnam (1996) revisaram a metodologia empregada e sugeriram que a DES avaliaria pelo menos duas categorias de experiência dissociativa: uma patológica e não dimensional (amnésia dissociativa, despersonalização, desrealização), e uma não patológica, mas dimensional (absorção/envolvimento imaginativo). A primeira categoria seria mais comum em populações clínicas, enquanto a segunda seria mais característica da população geral. Uma versão reduzida da escala com base nos itens não dimensionais foi chamada de DES-Taxon (Waller et al., 1996) e é considerada como mais susceptível ao rastreamento de dissociação patológica. Há, porém, controvérsias em relação ao caráter efetivamente mais patológico dos itens da DES-T, e também quanto à sua utilidade no diagnóstico diferencial dos transtornos dissociativos (Merrit \& You, 2008).

Embora não seja recomendado para fins diagnósticos, o escore de 30 da DES é considerado o ponto de corte acima do qual é possível rastrear pacientes portadores de algum transtorno dissociativo (Carlson \& Putnam, 1993). Em estudo feito por Fiszman, Cabizuca, Lanfredi, e Figueira (2004) durante o processo de tradução e adaptação transcultural da escala para o português do Brasil, $63 \%$ dos pacientes que apresentaram o escore acima de 30 e não apresentavam transtorno dissociativo de identidade tinham outro transtorno dissociativo ou transtorno de estresse pós-traumático. Todavia, de acordo com Carlson e Putnam (1993), um escore acima de 20 merece, por si só, uma investigação mais acurada do ponto de vista clínico, podendo também ser utilizado para identificar casos de tendência à dissociação. Já existem evidências sugerindo a nota de corte de 30 como sendo de utilidade para identificar casos de transtorno dissociativo e transtorno de crises não epiléticas psicogênicas em amostras brasileiras
(Fiszman et al., 2004; Negro, Palladino-Negro, \& Louzã, 2002; Proença, 2010).

A versão brasileira da DES também mostrou validade convergente com medidas de trauma infantil e estresse psicológico, especialmente aqueles itens que abarcam experiências dissociativas consideradas mais patológicas pela literatura internacional (Domingues Goi, 2012; Negro et al., 2002). Por sua vez, as pontuações na DES diminuíram em resposta à psicoterapia cognitivo-comportamental e ao tratamento farmacológico do transtorno de estresse pós-traumático (Lages et al., 2011; Lima et al., 2007), o que não só fornece validação adicional à escala, como também sugere que os dados obtidos no Brasil sobre a DES tendem a se comportar de modo compatível com o que já se sabe acerca das pesquisas estrangeiras.

$\mathrm{Na}$ versão original da DES (Bernstein \& Putnam, 1986), as respostas consistiam em assinalar, ao longo de uma escala visual analógica de 100 milímetros de comprimento, a frequência aproximada dos sintomas descritos. Os escores finais variavam de 0 a 100 , sendo 0 (nunca) e 100 (sempre). Em uma segunda versão, a mais recente da escala, denominada DES-II (Carlson \& Putnam, 1993) as respostas passaram a ser dadas em uma escala de porcentagem Likert, que também varia de 0 a 100, com diferentes intervalos pré-fixados. Na versão brasileira, os autores responsáveis pela tradução optaram por substituir o sistema de porcentagem devido à dificuldade dos respondentes com esse modelo de resposta, adotando-se então o mesmo sistema da versão para adolescentes (Smith \& Carlson, 1996), que é mais intuitivo, e cujas respostas variam apenas de 0 a 10 . Não se observou alteração, entretanto, na forma de obtenção dos escores, sendo preservada, assim, a equivalência operacional do instrumento (Fiszman et al., 2004).

Apesar dos estudos inventariados acima, a pesquisa da dissociação é ainda escassa no Brasil, comparativamente ao que tem sido feito em diversos outros países. O nível de esclarecimento sobre os transtornos dissociativos, sobre a aplicabilidade dessas categorias diagnósticas à população brasileira e sobre a prevalência das experiências dissociativas em amostras não clínicas é ainda bastante limitado, a despeito de alguns manuais e livros de psiquiatria básica e psicopatologia incluírem capítulos ou referências a esses transtornos (Alminhana \& Moreira-Almeida, 2011; Moreira-Almeida, Alvarado, \& Zangari, 2007). Uma vez que praticamente nenhum estudo brasileiro avaliou detidamente a consistência interna da versão brasileira da Escala de Experiências Dissociativas, bem como suas propriedades fatoriais e validade convergente, isto é, seu nível de correlação com outras variáveis psicológicas com as quais se espera que esteja correlacionada (como sintomas conversivos, somatização e trauma) e variáveis sociais e demográficas (a exemplo da idade, do sexo e das crenças e experiências religiosas) estudadas na literatura estrangeira, decidiu-se 
apresentar neste artigo os dados obtidos nesse sentido, a partir de uma pesquisa realizada com uma amostra não clínica de respondentes brasileiros.

O estudo (Maraldi, 2014), cujo banco de dados serviu de base para as análises psicométricas apresentadas neste artigo, teve como principal objetivo investigar, a partir de metodologia quali-quantitativa, em variadas frentes de coleta, as complexas relações existentes entre experiências dissociativas, crenças e experiências religiosas. Uma das motivações para a pesquisa surgiu do fato de que, historicamente, sintomas histéricos (dissociativos, somatoformes e conversivos) foram considerados pelos psicólogos e psiquiatras como a base de muitas experiências místicas e espirituais de transe (por exemplo, Janet, 1889). Tais crenças e experiências podem nos servir, assim, como úteis indicadores da presença de fenômenos dissociativos na população em geral, como já haviam sugerido Ross \& Joshi (1992). Elas estão também frequentemente correlacionadas com outras variáveis ligadas à dissociação, como sintomas depressivos e ansiógenos (Sharps, Matthews, \& Asten, 2008), queixas somáticas (Houran, Kumar, Thalbourne, \& Lavertue, 2002), trauma infantil (Berkowski \& MacDonald, 2014) e transliminaridade, uma medida de alterações de consciência subjacentes aos relatos de experiências religiosas e alegadamente paranormais (Thalbourne, 1998).

Por meio de questionários e escalas (frente quantitativa), a pesquisa procurou verificar se os padrões de correlações entre as variáveis supracitadas se manteriam em uma amostra brasileira e avaliou diferenças nas pontuações de participantes de diferentes afiliações religiosas e não religiosas. Mediante entrevistas e observações dos participantes, buscou-se avaliar o impacto dos fenômenos dissociativos na história de vida de respondentes religiosos, ateus e agnósticos e em rituais e práticas religiosas de transe, como sessões espíritas e grupos de oração carismáticos, visando identificar indivíduos mais predispostos às experiências dissociativas, bem como o eventual papel das práticas ritualísticas no manejo e modelagem desses sintomas (Maraldi, 2014).

A necessidade das questões sociodemográficas se justificou, em primeiro lugar, pelos poucos estudos sobre dissociação e variáveis relacionadas feitos no Brasil, de modo que tudo que se pudesse saber sobre a relação entre tais variáveis e indicadores sociodemográficos seria de relevância para o estudo da dissociação. Em segundo lugar, houve o fato de o estudo (Maraldi, 2014) ter tido como um de seus propósitos formular hipóteses psicossociais sobre as relações entre dissociação e experiências religiosas.

De modo a garantir maior confiabilidade para os resultados, tomou-se o cuidado de avaliar a estrutura fatorial e as propriedades psicométricas dos instrumentos antes de prosseguir com a análise dos dados, sendo que o principal teste empregado foi a Escala de Experiências Dissociativas. Para o presente artigo, são relevantes os dados quantitativos, em especial, aqueles obtidos por meio dessa escala. Para uma discussão sintética dos demais resultados e implicações da pesquisa, conferir Maraldi \& Zangari (2015).

\section{Método}

\section{Participantes}

Os participantes da frente quantitativa foram 1450 respondentes $(67,9 \%$ homens), de 18 anos de idade ou mais $(M=29,32 ; D P=11,27$, variação $=18-81$ anos $)$, que participaram de um survey online sobre dissociação e variáveis relacionadas, o qual serviu parcialmente de base para a tese de doutorado do primeiro autor (Maraldi, 2014). Embora a amostra citada seja de conveniência, cujos participantes foram obtidos por meio da divulgação do questionário on-line mediante a participação em redes sociais (Facebook e Orkut), mala direta (lista de contatos do pesquisador e de amigos ou conhecidos) e visitas a diferentes grupos religiosos para observação etnográfica, o questionário veio a se espalhar pela internet - como de ordinário ocorre nesse tipo de pesquisas - atingindo localidades as mais diversas do país, não obstante uma maior concentração de respondentes do Estado de São Paulo (41,51\% da amostra total). Além de participantes de 23 estados brasileiros e do Distrito Federal, houve quatro participantes brasileiros residentes no exterior.

\section{Instrumentos}

No intuito de investigar as relações entre dissociação, experiências religiosas e variáveis psicológicas associadas, outros instrumentos, além da Escala de Experiências Dissociativas, foram aplicados, incluindo:

O questionário sobre traumas na infância (Childhood Trauma Questionnaire - CTQ - Bernstein et al., 2003; Grassi-Oliveira, Stein, \& Pezzi, 2006), que consiste em 28 perguntas relacionadas a diferentes eventos traumáticos que compõem cinco subescalas: abuso físico, abuso emocional, abuso sexual, negligência física e negligência emocional. Além desses cinco componentes, o questionário inclui uma escala controle de minimização-negação das respostas. Foram realizados estudos de validação do instrumento com amostras brasileiras (Brodski, Zanon, \& Rutz, 2010; Seganfredo et al., 2009).

A escala revisada de crença paranormal (Revised Paranormal Belief Scale - RPBS - Tobacyk, 2004), que é considerada a mais comumente empregada em pesquisas sobre crenças paranormais. É constituída de 26 itens do tipo Likert com sete pontos que variam de discordo totalmente a concordo plenamente, os quais abarcam diferentes modalidades de crença paranormal e religiosa, como vida após a morte, reencarnação, ação direta da mente sobre a matéria, Deus, céu e inferno. A Escala de Crença Paranormal revisada foi traduzida e adaptada para português por membros do Laboratório de Psicologia Social da Religião do IP-USP, incluindo os dois autores do artigo. 
A escala de sintomas (Symptom Checklist-90-RevisedSCL-90-R - Derogatis, 1994) é uma escala multidimensional de autorrelato, de caráter diagnóstico, que avalia nove dimensões de sintomas, contabilizando um total de 90 itens cujos escores variam de 0 (nem um pouco) a 4 (muito) e foi traduzida, adaptada e validada para o português do Brasil (Carissimi, 2011; Laloni, 2001). Como a intenção era averiguar apenas os fenômenos somatoformes, bem como variáveis com as quais estivessem intimamente relacionados (a exemplo das queixas depressivas e ansiógenas), considerou-se para aplicação apenas as subescalas de Somatização, Depressão e Ansiedade. A primeira subescala avalia queixas ligadas aos sistemas cardiovascular, gastrointestinal e respiratório. Dores musculares e outras formas de desconforto corporal são também rastreadas. A subescala de Depressão registra sintomas de distúrbio do humor e da afetividade, como sinais variados de: retraimento, baixa autoestima, falta de motivação, pensamentos suicidas, etc. Já a última subsescala considerada, a de Ansiedade, mede sintomas como nervosismo, tremor, pânico e experiências semelhantes de medo ou terror (fóbicas). Dado que houve forte correlação entre os fatores e que a soma de todos os itens empregados denotou um alfa elevado $(0,954)$, decidiu-se utilizá-la como uma medida composta de sintomas psicossomáticos (somatização e seus correlatos).

Uma escala original de sintomas conversivos, doravante apelidada de ESC-7, foi elaborada, tendo como referência a literatura clássica e atual sobre fenômenos histéricos e conversivos. Essa escala de validade aparente (face-valid) foi desenvolvida para ser aplicada em conjunto com as subescalas da SCL-90-R, embora seguindo um modelo diferenciado de pontuação - cinco pontos que variam de nunca a sempre, o mesmo empregado para o questionário sobre traumas na infância. A ESC-7 aborda experiências conversivas clássicas, como episódio convulsivo, paralisia psicogênica, cegueira e surdez psicogênicas, alucinações visuais, desmaios e perda de consciência, "afonia histérica” e marcha instável (problemas de locomoção).

A Escala de Transliminaridade (Thalbourne, 1998), contendo 29 itens do tipo "verdadeiro" ou "falso". A escala avalia uma "hipotética tendência para que um conteúdo psicológico atravesse fronteiras dentro e fora da consciência” (Lange, Thalbourne, Houran, \& Storm, 2000 , p. 594). Tal definição abrangente inclui diversas alterações perceptivas e de consciência, geralmente relatadas em contextos religiosos, como vivências de absorção com a natureza, experiências místicas e paranormais e tendências excêntricas. A escala tem apresentado correlação positiva e significante com as variáveis de dissociação e crença paranormal e outros tipos de estado alterado de consciência vinculados a práticas religiosas e espirituais (Thalbourne, 1998).

A pesquisa também incluiu um extenso levantamento de dados sociais e demográficos dos participantes, abrangendo 1 . idade, 2. cidade onde reside, 3 . sexo (biológico), 4. estado civil, 5. orientação afetiva-sexual, 6. grupo étnico/racial, 7. coabitação, 8. ordem de nascimento, 9. renda mensal domiciliar, 10. nível de escolaridade, 11. disciplina em que se graduou (quando aplicável), 12. condição profissional atual, 13. profissão exercida (quando aplicável), 14. afiliação religiosa ou filosófica e 15. exposição a situações de violência social, estresse e discriminação. $O$ último item consistia em uma lista com 12 perguntas sobre experiências sociais traumáticas como ser "vítima de assalto à mão armada", ser "ameaçado de morte", "trabalhar em um emprego muito estressante" ou ser "vítima de piadas ofensivas e forte discriminação". As respostas variavam de "nunca" a "seis ou mais vezes".

\section{Procedimentos}

O projeto de pesquisa foi analisado e aprovado pelo Comitê de Ética em Pesquisa com Seres Humanos do Instituto de Psicologia da Universidade de São Paulo mediante o envio dos documentos pela Plataforma Brasil, CAAE: 09629712.0.0000.5561. A realização do projeto contou, ainda, com financiamento da Fundação de Amparo à Pesquisa do Estado de São Paulo ( ${ }^{\circ}$ do processo 2011/05666-1).

As escalas foram disponibilizadas pela internet (numa página específica da rede, via Google Docs), de forma didaticamente detalhada, tendo o preenchimento sido feito com comodidade pelos voluntários, dentro de um período previamente estipulado, e com retorno dos dados também pela internet. Várias versões do mesmo questionário foram elaboradas, cada qual com a ordem das questões modificada, no intuito de diminuir efeitos de ordem.

\section{Análise de Dados}

A compilação, a verificação de frequências e o cruzamento dos dados coletados foram feitos com o auxílio do Statistical Package for Social Sciences (SPSS), na sua versão 17 para Windows, e do programa Microsoft Excel, na versão 2010.

Em função das várias análises efetuadas e do número substancial de participantes, decidiu-se adotar um critério de significância estatística ligeiramente mais conservador $(p<0,01)$. A descrição das variáveis categóricas consistiu na apresentação das frequências de ocorrência e das porcentagens. As comparações entre as médias foram realizadas por meio da ANOVA de um fator. No caso de comparações não planejadas, foi utilizado o teste Post hoc de Games-Howell, devido às diferenças de tamanho entre os grupos e às diferenças de variância. Para avaliar o efeito de possíveis covariáveis na relação entre a variável dependente e a variável independente, empregou-se a ANCOVA. As análises correlacionais, por sua vez, basearam-se no coeficiente produto-momento de Pearson. Antes da análise dos dados, avaliou-se a estrutura fatorial e a consistência interna de cada instrumento empregado. 


\section{Resultados}

\section{Análise fatorial e consistência interna}

Apesar de a melhor solução fatorial em muitas pesquisas ter sido a solução de um único fator, alguns autores encontraram três subescalas além do escore total (Carlson et al., 1991; Ross et al.,1991). Contudo, considerando-se que as soluções fatoriais desses estudos se mostraram muitas vezes inconsistentes, foi preciso verificar como os dados se agrupariam na presente amostra. Inicialmente, foi observado $K M O=0,96$ e teste de Barlett significativo $(p<0,001)$, indicando que os dados estavam aptos para a análise de fatores.

Havia certa dúvida sobre qual o método mais adequado de extração, mas, nos vários delineamentos rodados (análise dos componentes principais, máxima verossimilhança, principais eixos fatoriais), os resultados obtidos foram, basicamente, os mesmos, com poucas variações. Todavia, a análise da distribuição dos dados da DES indicou se tratar de uma distribuição não normal, já que a maior parte das pessoas pontuou os valores mais baixos, o que é também consistente com os resultados das pesquisas estrangeiras envolvendo amostras não clínicas (Carlson \& Putnam, 1993; Ross, Joshi, \& Currie, 1990). Assim sendo, entendeu-se mais adequado apresentar os fatores extraídos pelo método dos principais eixos fatoriais. Essa análise revelou quatro componentes com eigenvalues maiores do que um - 11,78 (42\%); 1,59 $(5,6 \%) ; 1,23(4,4 \%) ; 1,08(3,8 \%)$, os quais, juntos, explicavam $56 \%$ da variância total. Optou-se por uma rotação oblíqua (oblimin) pela expectativa de correlação entre os fatores. Cargas inferiores a 0,30 foram excluídas. Para a retenção dos fatores, tomou-se por base, inicialmente, o critério de Kaiser e a coerência e significância conceitual.

O quarto componente dessa primeira rotação (formado por três itens de absorção) não fazia muito sentido teórico, e dois dos itens que o compunham carregavam moderadamente também em outros fatores. A solução trifatorial, ao contrário, fez total sentido teórico, replicando as subescalas encontradas em algumas pesquisas internacionais, e a consistência interna foi bastante satisfatória, como se verá adiante.

Em consideração às críticas de alguns autores de que o critério de Kaiser-Gutman, tende a superestimar o número de fatores a ser retido devido ao erro amostral (Damásio, 2012), decidiu-se recorrer, adicionalmente, ao método da análise paralela, conhecido por ser um método de extração menos arbitrário, o qual faz uso da simulação de dados randômicos com o mesmo tamanho amostral e número de variáveis (no caso do nosso estudo, 28 variáveis/itens da DES para 1450 participantes) no intuito de estimar a quantidade de fatores a ser extraída (Hayton, Allen, \& Scarpello, 2004). Os fatores extraídos dos dados empíricos cujos eigenvalues forem maiores que os correspondentes eigenvalues dos dados aleatórios são os que devem ser retidos.

Seguindo as recomendações de O'Connor (2000), para realização do teste por meio do SPSS, conduziu-se uma análise dos componentes principais com permutação dos dados brutos, a partir da simulação de 1000 repetições de conjuntos de dados e percentil de $95 \%$. Os resultados obtidos sustentaram a solução de apenas três fatores (sugerida antes pelo método de Kaiser e significância teórico-conceitual): eigenvalues empíricos $=11,782$, 1,590, 1,237, 1,087 ; eigenvalues médios $=1,261,1,226,1,198,1,175 \ldots$; eigenvalues percentis $=1,296,1,253,1,221,1,196$.

$\mathrm{Na}$ Tabela 1, é possível conferir as cargas para cada fator, a nomeação dada a cada um e os itens que os compõem, bem como dados de consistência, correlações entre os fatores e as médias obtidas para cada subescala. O fator absorção envolve experiências de envolvimento imaginativo, distraibilidade e insensibilidade à dor. $\mathrm{O}$ fator despersonalização/desrealização abarca experiências de alteração da identidade e da percepção do ambiente. O fator amnésia inclui questões sobre distúrbios diversos de memória frequentemente associados a fenômenos dissociativos.

A consistência interna da versão brasileira da DES foi bastante satisfatória. Para essa avaliação, utilizou-se o alpha de Cronbach total e subescalas (conferir Tabela 1), e medidas adicionais fornecidas pelo SPSS para o delineamento das duas metades (Split-half), como os coeficientes de Guttman $(0,889)$ e Spearman-Brown $(0,909)$. A correlação entre as duas metades da escala (14 itens cada) foi de 0,834 , com os respectivos valores de alpha: 0,891 (primeira metade) e 0,913 (segunda metade). Os fatores também obtiveram forte correlação entre si e com o escore total. Para o escore dessas subescalas, foram empregadas as médias dos participantes da pesquisa. Calculouse, também, o escore total da DES, conforme instruções de Fiszman et al. (2004). Tal como observado em outras pesquisas com amostras não clínicas (Irwin, 1999), os itens de absorção obtiveram média maior que as demais subescalas da DES.

Tabela 1

Estrutura Fatorial, Alpha de Cronbach, Médias na Amostra Estudada e Correlações entre os Fatores da DES

\begin{tabular}{lccccc}
\hline \multicolumn{3}{c}{ Fatores/Nomeações } & & & \\
\hline & \multirow{2}{*}{ Escore total } & $(1)$ & & $(2)$ & $(3)$ \\
Absorção & Despersonalização & Amnésia \\
\hline$\alpha$ & & 0,945 & 0,890 & 0,857 & 0,884 \\
$M ; D P$ & $19,13 \pm 16,15$ & $3,06 \pm 2,07$ & $1,12 \pm 1,66$ & $1,20 \pm 1,50$ \\
\hline
\end{tabular}


Tabela 1 (continuação)

Estrutura Fatorial, Alpha de Cronbach, Médias na Amostra Estudada e Correlações entre os Fatores da DES

\begin{tabular}{lcccc}
\hline & Correlações entre os fatores & & \\
\hline Escore total & - & 0,942 & 0,863 & 0,911 \\
Absorção & - & - & 0,712 & 0,768 \\
Despersonalização & - & - & - & 0,743 \\
Amnésia & - & - & - & - \\
\hline
\end{tabular}

1. Não lembrar-se de toda ou parte da via-

Itens e cargas fatoriais gem (carro, ônibus, metrô).

0,376

2. Não ouvir toda ou parte da conversa .

0,568

3. Não saber como chegou a um lugar.

0,471

4. Roupas que não lembra ter colocado.

5. Objetos novos entre suas coisas.

6. Abordado por pessoas que não lembra ter conhecido. pessoa.

8. Não reconhecer amigos ou familiares.

9. Não lembrar de eventos importantes de sua vida.

10. Ser acusado de mentir.

11. Olhar no espelho e não se reconhecer.

12. Pessoas e coisas são irreais.

13. Corpo não me pertence.

14. Recordações vívidas.

15. Confundir sonho e realidade.

0,674

16. Não reconhecer lugar conhecido.

17. Envolver-se profundamente com histó0,726 rias em televisão ou filmes.

18. Sonhar acordado.

0,600

19. Ser capaz de não sentir dor.

0,318

20. Não perceber a passagem do tempo.

0,728

21. Falar sozinho.

0,613

22. Ser como duas pessoas diferentes.

23. Ser capaz, em algumas situações, de

0,419 fazer com facilidade o que normalmente é difícil.

24. Dúvida sobre se fez ou apenas pensou em fazer.

25. Evidências de coisas que não lembra ter feito.

26. Papéis, desenhos ou notas que não lembra ter feito.

27. Vozes dentro da cabeça. 


\section{Variáveis sociais e demográficas}

Não houve correlação significativa entre o escore total da DES e idade, exceto para a subescala de absorção $(r=-0,147, p<0,001)$. No escore total, as mulheres $(M=21,12 ; \quad D P=17,26)$ pontuaram significativamente acima dos homens $(M=18,20 ; D P=15,53), t(1448)=-$ 3,$23 ; p=0,001$, o que se confirmou para as subescalas de absorção e despersonalização, mas não para a de amnésia, $t(1448)=0,80 ; p=0,235$. Diversas outras variáveis da pesquisa, incluindo escolaridade e habitação diferiram em função do gênero e da idade. Controlando os efeitos dessas variáveis em análises estatísticas subsequentes por meio de análise de covariância, descobriu-se que os que moram sozinhos $(M=22,20 ; D P=18,36)$ pontuaram acima na DES do que os que não moram $(M=18,82$; $D P=15,89), F(1,1446)=8,08 ; p=0,005$. Houve também diferença no nível de dissociação conforme a escolaridade, $F(7,1440)=3,17 ; p=0,002$. Observou-se que participantes com ensino fundamental completo obtiveram média maior que os participantes do ensino médio $(p=0,006)$ e da pós-graduação $(p=0,002)$; os respondentes com ensino médio completo pontuaram acima $(\mathrm{p}=0,001)$ dos pós-graduandos; os pós-graduandos $(p=0,003)$ pontuaram abaixo dos que possuem o ensino superior completo.

A DES também correlacionou positiva e significativamente (mas de maneira fraca) com a frequência de visitas à psicoterapia ou ao psiquiatra $(r=0,165, p<0,001)$, e com a exposição a vivências de ameaça de morte $(r=0,125, p<0,001)$ e discriminação social $(r=0,126$, $p<0,001$ ), mesmo controlando os efeitos do gênero por meio de correlação parcial (homens e mulheres haviam significativamente diferido nas duas primeiras variáveis). Membros de grupos religiosos $(M=23,00, D P=18,54)$ e pessoas sem afiliação religiosa ou filosófica definida $(M=23,57, D P=18,77)$ pontuaram significativamente acima, $F(2,1445)=32,60, p<0,001$, dos ateus e agnósticos $(M=17,28, D P=14,55)$. Esse resultado se manteve nas diferentes subescalas da DES. A média mais alta foi a dos membros de círculos esotéricos e ocultistas $(M=26,20, D P=18,03)$.

\section{Evidências de validade convergente}

$\mathrm{Na}$ Tabela 2 é possível observar as correlações entre a DES e os principais instrumentos do estudo. Tomouse o cuidado de controlar os efeitos do gênero e da idade (correlações parciais), considerando-se os resultados anteriores acerca das variáveis sociodemograficas. A DES correlacionou positiva e significantemente com instrumentos com os quais se esperava que viesse a correlacionar, como a medida composta de sintomas psicossomáticos (somatização, ansiedade e depressão combinadas), a escala de sintomas conversivos, a crença paranormal e religiosa e a escala de transliminaridade (uma medida de alterações perceptivas e de consciência relatadas em contextos religiosos). $\mathrm{O}$ mesmo padrão de correlações foi observado para todas as subescalas da DES.

Tabela 2

Correlações de Pearson entre a DES e as Principais Escalas da Pesquisa

\begin{tabular}{cccccccccc}
\hline & RPBS & TS & ESC-7 & SCLPSICO $^{1}$ & PN & EN & EA & PA & SA \\
\hline DES & $0,325^{*}$ & $0,532^{*}$ & $0,371^{*}$ & $0,446^{*}$ & $0,112^{*}$ & $0,118^{*}$ & $0,207^{*}$ & $0,167^{*}$ & $0,157^{*}$ \\
\hline
\end{tabular}

Nota. *Correlações significativas no nível de $p<0,001$. DES=Escala de Experiências Dissociativas; RPBS=Escala Revisada de Crença Paranormal; SCLPsico.=Medida composta de sintomas psicossomáticos extraídos da escala de sintomas (somatização, depressão e ansiedade); ESC-7=Escala de Sintomas Conversivos. TS=Escala de Transliminaridade; PN=Negligência Física; EN=Negligência Emocional; EA=Abuso Emocional; PA=Abuso Físico; $\mathrm{SA}=\mathrm{Abuso}$ Sexual.

${ }^{1}$ Das subescalas da SCL, a dissociação correlacionou positivamente com todas (somatização, $r=0,393$, depressão, $r=0,387$, ansiedade, $r=0,421)$

Tomando-se a amostra total da pesquisa, houve diferenças significativas entre os que pontuaram acima e abaixo da nota de corte de 30 na DES em relação a experiências de abuso emocional, $F(1,1447)=38,35$, $p<0,001$, abuso físico, $F(1,1447)=21,33, p<0,001 \mathrm{e}$ abuso sexual, $F(1,1447)=11,70, p=0,001 \quad$ (conferir Tabela 3 ). Esses resultados se mantiveram mesmo controlando os efeitos do gênero. Não houve diferenças significativas para as subescalas de negligência física e emocional.

Tabela 3

Médias e Desvio Padrão dos Participantes para as Subescalas de Abuso do CTQ

\begin{tabular}{lcc}
\hline & DES $<30$ & DES $\geq 30$ \\
\hline Abuso emocional & $8,91 \pm 3,99$ & $10,70 \pm 4,81$ \\
Abuso físico & $6,91 \pm 2,71$ & $7,81 \pm 3,73$ \\
Abuso sexual & $5,71 \pm 2,19$ & $6,34 \pm 3,62$ \\
\hline
\end{tabular}

Nota. CTQ=Questionário sobre Traumas na Infância; DES=Escala de Experiências Dissociativas 


\section{Discussão}

Este é o primeiro estudo de que os autores têm conhecimento a se utilizar da DES em uma amostra grande de respondentes brasileiros, apesar de não representativa da população. As evidências encontradas indicam a validade da versão brasileira da DES. Em primeiro lugar, sua ótima consistência interna e a estrutura fatorial condizente com as variáveis avaliadas por esse instrumento e com outras soluções fatoriais encontradas em pesquisas estrangeiras (Carlson et al., 1991; Ross et al., 1991). Em segundo lugar, sua correlação com as variáveis esperadas (validade convergente) como os sintomas conversivos e de somatização e as experiências traumáticas (incluindo o fato de pessoas com escores iguais ou acima de 30 terem apresentado média significativamente maior em abuso emocional, abuso físico e abuso sexual, mesmo controlando os efeitos da variável sexo).

Esses vários resultados parecem sustentar a ideia de que, a despeito da variação no procedimento de pontuação dos itens, a DES brasileira tem se comportado, em muitos aspectos, de modo bastante semelhante às versões desenvolvidas em outros países (Carlson \& Putnam, 1993). Ademais, como Carlson e Putnam (1993) já haviam salientado, mudanças nos itens ou na forma de pontuação são esperadas e bem-vindas, sempre no intuito de se assegurar melhor adaptação a uma determinada cultura.

As correlações significativas da DES com as Escalas de Crença Paranormal e Transliminaridade (uma medida de alteração da consciência e alterações perceptivas interpretadas como espirituais) parecem confirmar pesquisas anteriores que associavam a dissociação aos relatos de experiências alegadamente místicas e religiosas (Irwin, Schofield, \& Baker, 2014; Thalbourne, 1998). Talvez seja importante mencionar que as pontuações em trauma infantil não diferiram entre os grupos de religiosos e não religiosos (Maraldi, 2014). É provável, assim, que boa parte da dissociação vivida nesses contextos tenha origem distinta daquela ocasionada por processos patológicos derivados de experiências traumáticas. Como visto antes, os membros de círculos esotéricos e ocultistas (como integrantes da Rosacruz ou da Teosofia) foram os que obtiveram a maior média na DES, talvez em função de práticas que estimulam alterações de consciência e absorção imaginativa (como meditação, técnicas de visualização, etc.), ou devido ao fato de indivíduos mais propensos à dissociação procurarem mais por esses contextos.

As correlações positivas da DES com sintomas conversivos e psicossomáticos, apesar de não elevadas, sustentam uma aproximação entre dissociação cognitiva e somatoforme, mesmo para o contexto de uma amostra não clínica. Todavia, deve-se salientar que, uma vez que nenhum exame médico foi realizado com os respondentes, é possível que parte de suas pontuações em sintomas físicos se relacione a causas não psicogênicas (muito embora o questionário contivesse instruções para que o participante excluísse causas orgânicas identificadas).

As mulheres pontuaram significantemente acima dos homens, não só em absorção e despersonalização como em outras medidas, incluindo traumas na infância (Maraldi, 2014), corroborando outras pesquisas nesse sentido (Born, Phillips, Steiner, \& Soares, 2005; Irwin, 1999). Em nosso estudo, a correlação negativa da DES com a idade se confirmou apenas para a subescala de absorção, o que talvez signifique que pessoas mais jovens tendem a fantasiar e a imaginar mais, embora não necessariamente difiram dos mais velhos em outras dimensões da dissociação.

Apesar de os participantes com escores mais elevados na DES terem relatado com maior frequência certas formas de trauma, as correlações entre a DES e as diferentes subescalas do questionário sobre traumas na infância foram fracas, o que sugere que outros fatores podem estar envolvidos na associação entre dissociação e trauma. O fato de se tratar de amostra não clínica poderia explicar, em parte, tais resultados, uma vez que a média de procura por atendimento psicológico ou psiquiátrico foi menor que uma visita $(M=0,53$; $D P=0,89$, Moda $=0$ ). A média em absorção foi maior do que em despersonalização e amnésia, sendo que estas duas últimas modalidades são consideradas, geralmente, mais patológicas e mais relacionadas a uma etiologia traumática (Nijenhuis \& Van der Hart, 2011). De qualquer maneira, a DES também correlacionou com situações de ameaça de morte e discriminação social, o que indica que a relação entre dissociação e vivências traumáticas diversas possui alguma consistência com base nos resultados.

Um dado inesperado foi a diferença entre os que moram sozinhos e os que moram acompanhados no escore total da DES, o que indica que pessoas sozinhas tendem a relatar mais experiências dissociativas. O relativo isolamento experimentado nos instantes de solidão talvez predisponha esses indivíduos a vivências imaginativas e de absorção, ou mesmo a outras vivências insólitas (por exemplo, ilusões e alucinações), as quais, aliás, são típicas em sujeitos mantidos em cativeiro, presos ou afastados por longo tempo do mundo externo e do convívio social (West \& Martin, 1994). É bem possível que pessoas que residem sozinhas experimentem, de tempos em tempos, vivências dessa natureza, embora com menor intensidade do que sujeitos que foram isolados involuntariamente e sob forte pressão psicológica. Outra explicação possível é a de que ao menos parte das pessoas que moram sozinhas apresentariam características de personalidade esquiva, ou talvez traços de leve excentricidade (por exemplo, esquizotipia) que não apenas tornariam o convívio com outra pessoa mais difícil como também as levaria a experimentarem maiores níveis de dissociação. 
Viu-se, anteriormente, que participantes com menor escolaridade pontuaram mais na DES, o que pode estar relacionado ao nível de leitura e compreensão (Van Ijzendoorn \& Schuengel, 1996). Mas talvez uma explicação para isso resida no fato de que, tendo menos acesso aos estudos, tais indivíduos teriam apresentado menos chances de desenvolver certas capacidades intelectuais ou mnêmicas, o que se traduziria em (ou expressaria) maior número de falhas cognitivas (falhas de memória, dificuldade de concentração, etc.), maior tendência a confabular e imaginar (em oposição ao pensamento lógico) ou menor capacidade de expressão e comunicação de ideias. Mais estudos são necessários para aclarar esse resultado.
A presente pesquisa forneceu evidências de validade relevantes acerca da versão brasileira da Escala de Experiências Dissociativas. Investigações futuras deverão considerar o emprego de técnicas de análise fatorial confirmatória, bem como realizar comparações entre amostras clínicas e não clínicas (preferencialmente representativas dessas populações). Avaliações sistemáticas da utilidade dessa escala para rastreio dos transtornos dissociativos deverão ser igualmente conduzidas, incluindo avaliações da chamada DES-Taxon, uma versão reduzida com base nos itens considerados mais susceptíveis ao rastreamento de dissociação patológica (Waller et al., 1996).

\section{Referências}

Alminhana, L. O., \& Moreira-Almeida, A. (2011). Transtornos da personalidade e transtornos dissociativos. Em M. R. Louzã Neto (Ed.). Transtornos da personalidade (pp. 189-204). Porto Alegre: Artmed.

Alvarado, C. S. (2005). Research on non-pathological dissociation. Ciencias de la Conducta, 20(1), 31-56.

American Psychiatric Association (2013). Diagnostic and statistical manual of mental disorders: DSM-5. Washington, DC: American Psychiatric Publishing.

Bernstein, E. M., \& Putnam, F. W. (1986). Development, reliability and validity of a dissociation scale. Journal of Nervous and Mental Disease, 174, 727-735.

Bernstein, D. P., Stein, J. A., Newcomb, M. D., Walker, E., Poqqe, D., Ahluvalia, T., ... Zule, D. (2003). Development and validation of a brief screening version of the childhood trauma questionnaire. Child Abuse \& Neglect, 27, 169-190.

Berkowski, M., \& MacDonald, D. A. (2014). Childhood trauma and the development of paranormal beliefs. Journal of Nervous and Mental Disease, 202(4), 305-312.

Born, L., Phillips, S. D., Steiner, M., \& Soares, C. N. (2005). Trauma e o ciclo reprodutivo feminino. Revista Brasileira de Psiquiatria, 27, 65-62.

Brodsky, S. K., Zanon, C., \& Hutz, C. S. (2010). Adaptação e validação do questionário para traumas na infância (QUESI) em uma amostra não clínica. Avaliação Psicológica, 9(3), 499-501.

Cardeña, E., \& Carlson, E. (2011). Acute stress disorder revisited. Annual Review of Clinical Psychology, 7, 245-267.

Carlson, E. B., \& Putnam, F. W. (1993). An update on the dissociative experiences scale. Dissociation, 6(1), 16-27.

Carlson E. B., Putnam, F. W., Ross, C. A., Anderson, G., Clark, P., \& Torem, M. (1991). Factor analysis of the dissociative experiences scale: A multicenter study. Em B. G. Braun. \& E. B. Carlson (Eds). Proceedings of the Eighth International Conference on Multiple Personality and Dissociative States (p. 16). Chicago: Rush.

Carissimi, A. (2011). Examinando fatores causais de sintomas psicológicos através do SCL-90-R em pacientes com apneia do sono grave (Dissertação de mestrado não publicada). Universidade Federal do Rio Grande do Sul, Porto Alegre, RS, Brasil.

Dalenberg, C. J., Brand, B. L., Gleaves, D. H., Dorahy, M. J., Loewenstein, R. J., ... Spiegel, D. (2012). Evaluation of the evidence for the trauma and fantasy models of dissociation. Psychological Bulletin, 138(3), 550-588.

Dalenberg, C. J., Brand, B. L., Gleaves, D. H, Dorahy, M. J., Loewenstein, R. J., Cardeña E., ... Spiegel, D. (2014). Reality versus fantasy: Reply to Lynn et al. (2014). Psychological Bulletin, 140(3), 911-920.

Damásio, B. F. (2012). Uso da análise fatorial exploratória em psicologia. Avaliação Psicológica, 11(2), 213-228.

Derogatis, L. R. (1994). Symptom Checklist-90-R (SCL-90-R). Administration, scoring and procedures manual. Minneapolis: National Computer Systems, USA.

Domingues Goi, J. (2012). Da dissociação à resiliência: a influência das experiências infantis no estilo de defesa em adultos (Dissertação de mestrado não publicada). Universidade Federal do Rio Grande do Sul, Porto Alegre, RS, Brasil.

Fiszman, A., Cabizuca, M., Lanfredi, C., \& Figueira, I. (2004). A adaptação transcultural para o português do instrumento Dissociative Experiences Scale para rastrear e quantificar os fenômenos dissociativos. Revista Brasileira de Psiquatria, 26(3), 164-173.

Grassi-Oliveira, R., Stein, L. M., \& Pezzi, J. C. (2006). Tradução e validação de conteúdo da versão em português do Childhood Trauma Questionnaire. Revista de Saúde Pública, 40(2), 249-255.

Hayton, J. C., Allen, D. G., \& Scarpello, V. (2004). Factor retention decisions in exploratory factor analysis: A tutorial on parallel analysis. Organizational Research Methods, 7(2), 191-205.

Houran, J., Kumar, V. K., Thalbourne, M. A., \& Lavertue, N. E. (2002). Haunted by somatic tendencies: Spirit infestation as psychogenic illness. Mental health, religion and culture, 5(2), 119-133.

Irwin, H. J. (1999). Pathological and nonpathological dissociation: The relevance of childhood trauma. The Journal of Psychology, 13(2), 157-164.

Irwin, H. J., Schofield, M. B., \& Baker, I. S. (2014). Dissociative tendencies, sensory-processing sensitivity and aberrant salience as predictors of anomalous experiences and paranormal attributions. Journal of the Society for Psychical Research, 78(917), 193-206. 
Janet, P. (1889). L'automatisme psychologique: Essai de psychologie expérimentale sur les formes inférieures de l'activité humaine. Versão eletrônica para a coleção "Les classiques des sciences sociales". Québec (Chicoutimi): L'Université du Québec à Chicoutimi.

Jepsen, E. K. K., Langeland, W., \& Heir, T. (2014). Early traumatized inpatients high in psychoform and somatoform dissociation: Characteristics and treatment response. Journal of Trauma and Dissociation, 15(5), 572-587.

Kihlstrom, J. F. (1994). One hundred years of Hysteria. Em S. J. Lynn \& J. W. Rhue (Ed.). Dissociation: Clinical and theoretical perspectives (pp. 365-394). New York: Guilford Press.

Krippner, S. (1997). The varieties of dissociative experience. Em S. Krippner \& S. M. Powers (Eds.). Broken images, broken selves: Dissociative narratives in clinical practice (pp. 336-361). Washington (DC): Brunner/Mazel.

Kruesi, M. E., Borckhardt, J. J., Younger, J., Nash, M. R., \& Shaw, D. (2004). Perceived links between physical problems and stress may be clouded by dissociative processes. Journal of Trauma and Dissociation, 5(4), 121-127.

Lages, A. C., Nórte, C. E., Pedrozo, A. L., Gonçalves, R. M., Marques-Portella, C., Souza, G. G. L., ... Ventura, P. R. (2011). Marcadores neurobiológicos e psicométricos da eficácia da terapia cognitivo-comportamental no transtorno de estresse pós-traumático associado a sintomas dissociativos: relato de caso. Revista de Psiquiatria do Rio Grande do Sul, 33(1), 63-67.

Laloni, D. T. (2001). Escala de avaliação de sintomas-90-R (SCL-90-R): adaptação, precisão e validade (Tese de doutorado não publicada). Pontífica Universidade Católica de Campinas, Campinas, Brasil.

Lange, R., Thalbourne, M. A., Houran, J., \& Storm, L. (2000). The revised transliminality scale: Reliability and validity data from a Rasch top-down purification procedure. Consciousness and Cognition, 9, 591-617.

Lima, A. A., Fiszman, A. Portella, C. M., Almeida, Y. A., Salomão, F. P., Geoffroy, R. M. G., \& Figueira, I. (2007). Negligência das classificações diagnósticas atuais com os fenômenos dissociativos do transtorno de estresse pós-traumático. Revista de Psiquiatria Clínica, 34(3), 139-143.

Lynn, S. J., Lilienfeld, S. O., Merckelbach, H., Giesbrecht, T., McNally, R. J., Loftus, E. F., ... Malaktaris. A. (2014). The trauma model of dissociation: Inconvenient truths and stubborn fictions. Comments on Dalenberg et al. (2012). Psychological Bulletin, 140(3), 896-910.

Maraldi, E. O. (2014). Dissociação, crença e identidade: uma perspectiva psicossocial (Tese de doutorado não publicada). Universidade de São Paulo, São Paulo, Brasil.

Maraldi, E. O., \& Zangari, W. (2015). "Em transe”: um estudo quali-quantitativo sobre o papel das experiências dissociativas e somatoformes nas crenças e rituais religiosos. Boletim Academia Paulista de Psicologia, 35(89), 382-408.

Merrit, R. D., \& You, S. (2008). Is there really a dissociative taxon on the dissociative experiences scale? Journal of Personality Assessment, 90(2), 201-203.

Moreira-Almeida, A., Alvarado, C., \& Zangari, W. (2007). Transtornos dissociativos (ou conversivos). Em M. R. Louzã Neto \& H. Elkis (Ed.). Psiquiatria Básica. (2 Ed. pp. 285-297). Porto Alegre: Artmed,

Negro, P. J. Palladino-Negro, P., \& Louzã, M. R. (2002). Do religious mediumship dissociative experiences conform to the sociocognitive theory of dissociation? Journal of Trauma and Dissociation, 3(1), 51-73.

Nijenhuis, E. R. S. (2000). Somatoform dissociation: Major symptoms of dissociative disorders. Journal of Trauma and Dissociation, 1(4), 7-32.

Nijenhuis, E. R. S. (2014). Dissociation in the DSM-5: Your view S'Il vous plaît, docteur Janet? Journal of Trauma and Dissociation, 15(3), 245-253

Nijenhuis, E. R. S., \& Van der Hart, O. (2011). Defining dissociation in trauma. Journal of Trauma and Dissociation, 12(4), 469-473.

O'Connor, B. P. (2000). SPSS and SAS programs for determining the number of components using parallel analysis and Velicer's MAP test. Behavior Research Methods, Instruments \& Computer, 32(3), 396-402.

Proença, I. C. G. F. (2010). Estudo sobre história de trauma e eventos dissociativos em pacientes com crises não epilépticas psicogênicas (Dissertação de mestrado não publicada). Universidade de São Paulo, São Paulo, Brasil.

Ross, C. A., Joshi, S., \& Currie, R. (1990). Dissociative experiences in the general population. American Journal of Psychiatry, 147(11), 15471552.

Ross, C. A., Joshi, S., \& Currie, R. (1991). Dissociative experiences in the general population: A factor analysis. Hospital and Community Psychiatry, 42(3), 297-301.

Ross, C. A., \& Joshi, S. (1992). Paranormal experiences in the general population. Journal of Nervous and Mental Disease, 180(6), $357-361$.

Seganfredo, A. C. G., Torres, M., Salum, G. A., Blaya, C., Acosta, J., Eizirik, C., \& Manfro, G. G. (2009). Gender differences in the association between trauma and parental bonding in panic disorder. Revista de Psiquiatria Clínica, 3(4), 314-321.

Sharps, M. J., Matthews, J., \& Asten, J. (2006). Cognition and belief in paranormal phenomena: Gestalt/feature-intensive processing theory and tendencies toward ADHD, depression and dissociation. The Journal of Psychology, 140(6), 579-590.

Simms, L. J., Prisciandaro, J. J., Krueger, R. F., \& Goldberg, D. P. (2012). The structure of depression, anxiety and somatic symptoms in primary care. Psychological Medicine, 42(1), 15-28.

Smith, S. R., \& Carlson, E. B. (1996). Reliability and validity of the adolescent dissociative experiences scale. Dissociation, 9(2), 125-129.

Spitzer, C., Barnow, S., Freyberger, H. J., \& Grabe, H. J. (2006). Recent developments in the theory of dissociation. World Psychiatry, 5(2), $82-86$.

Thalbourne, M. A. (1998). Transliminality: Further correlates and a short measure. Journal of the American Society of Psychical Research, 92, 402-419.

Tobacyk, J. J. (2004). A revised paranormal belief scale. The International Journal of Transpersonal Studies, 23(2), 94-98.

Van Ijzendoorn, M. H., \& Schuengel, C. (1996). The measurement of dissociation in normal and clinical populations: Meta-analytic validation of the Dissociative Experiences Scale (DES). Clinical Psychology Review, 16(5), 365-382.

Van der Hart, O., \& Dorahy, M. J. (2009). History of the concept of dissociation. Em P. F. Dell \& J. A. O’Neil (Eds.). Dissociation and the dissociative disorders: DSM-V and beyond (pp. 3-26). New York: Routledge.

Waller, N. G., Carlson, E. B., \& Putnam, F. W. (1996). Types of dissociation and dissociative types: A taxometric analysis of dissociative experiences. Psychological Methods, 1(3), 300-321. 
West, L. J., \& Martin, P. (1994). Pseudo-identity and the treatment of personality change in victims of captivity and cults. Em S. J. Lynn \& J. R. Rhue (Ed.). Dissociation: clinical and theoretical perspectives (pp; 268-288). New York: Guilford Press.

\section{Sobre os autores}

Everton de Oliveira Maraldi é graduado em psicologia pela Universidade Guarulhos. Mestre e doutor em psicologia social pelo Instituto de Psicologia da USP. Atualmente, trabalha como pesquisador de pós-doutorado na USP, com financiamento da FAPESP.

Wellington Zangari é graduado em psicologia pela Universidade Paulista, mestrado em ciências da religião pela Pontifícia Universidade Católica de São Paulo, doutorado em psicologia social pela Universidade de São Paulo e pós-doutorado em psicologia social pela USP, com estágio na Division of Personality Studies - University of Virginia. É professor doutor do Departamento de Psicologia Social e do Trabalho do Instituto de Psicologia da USP. 